\title{
Thiourea supplementation reduces arsenic accumulation in two selected rice (Oryza sativa L.) cultivars in a field study in Bengal Delta Basin, India
}

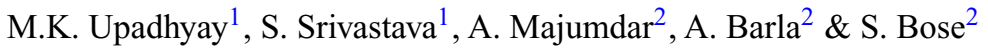 \\ ${ }^{1}$ Institute of Environment and Sustainable Development, Banaras Hindu University, Varanasi, U.P., India \\ ${ }^{2}$ Earth and Environmental Science Research Laboratory, Department of Earth Sciences, Indian Institute of Science \\ Education and Research Kolkata (IISER-K), Mohanpur, West Bengal, India
}

\begin{abstract}
Arsenic (As) accumulation in rice is a severe problem in Southeast Asia including West Bengal, India. Thiourea (TU) is a redox active thiol (-SH) based molecule, which has been found to regulate redox equilibrium and As level in lab study. In this work, we, for the first time, evaluated the effect ofTU supplementation on growth and yield of rice plants, biochemical changes and As concentrations in rice grains. Both rice cultivars (Satabdi-IET4786 and Gosai) were grown in three fields (Control, Low As and High As) in Nadia, West Bengal. The result indicated positive impact of TU supplementation on rice growth and yield and most importantly, showed reduction in As concentrations in rice grains.
\end{abstract}

\section{INTRODUCTION}

Arsenic (As) is well known class 1 carcinogen classified by IARC. Presently, 150 million people around the world are affected by As hazard. The condition is severe in Bengal delta basin region. There are many districts in West Bengal, where groundwater As concentration is $>50 \mu \mathrm{g} \mathrm{L}^{-1}$ including Nadia. This groundwater is used for irrigation in rice fields throughout the concerned area. Therefore, rice accumulates As in significant concentrations due to its favorable flooded cultivation, suitable transporters (channel proteins) and reducing environment. Being a staple crop worldwide, rice is the major source of As exposure to humans. Sulphur deficiency, redox imbalance, induced generation of reactive oxygen species (ROS) are key symptoms of As toxicity. Sulfur is important for plants as it affects As uptake, translocation and accumulation in rice plants (Srivastava et al., 2007). TU is sulfur containing redox regulator and ROS scavenger, which has already applied in variety of stresses in plants (Kelner et al., 1990). We applied $\mathrm{TU}$ and assessed its impacts on As accumulation in rice grains, yield, growth and antioxidant defense system.

\section{METHODS/EXPERIMENTAL}

\subsection{Site description}

The study was conducted in Sarapur, Dewali Gram panchayat $23^{\circ} 01^{\prime} 07.8^{\prime \prime} \mathrm{N} 88^{\circ} 39^{\prime} 43.1^{\prime \prime} \mathrm{E}-23^{\circ} 01^{\prime} 14.3^{\prime \prime} \mathrm{N}$ $88^{\circ} 38^{\prime} 24.7^{\prime \prime} \mathrm{E}$, Chakdaha block in Nadia district of West Bengal, India. This area is known for prolonged use of As contaminated groundwater for irrigation purposes. Here agriculture is the primary profession of the native people and so, this region may act as representative site for agriculture based As study.

\subsection{Growth of plants, collection and processing the samples}

We choose two rice genotypes (Satabdi-IET4786 and Gosai), which are the local and predominant variety being used in the study area. The cultivation of rice was performed in Monsoon (Aman, July-October 2016) in three selected fields vizLow As $\left(38.74 \pm 0.13 \mathrm{mg} \mathrm{kg}^{-1}\right)$, High As $(84.77 \pm$ $\left.0.51 \mathrm{mg} \mathrm{kg}^{-1}\right)$ and Control $\left(22.15 \pm 0.2 \mathrm{mg} \mathrm{kg}^{-1}\right)$ with/without thiourea (TU) application $\left(500 \mu \mathrm{g} \mathrm{L}^{-1}\right.$ for seed soaking and foliar application at the flowering stage). The area of per selected field was 400 square feet. The plant samples were taken to lab preserved in ice filled box. Plants were analyzed for pigment and antioxidant enzyme analyses after crushing in liquid $\mathrm{N}_{2}$. The remaining plants were washed thrice, air dried after evaluating length, tiller no. and other growth parameter. Further, they were separated into different parts i.e. root, straw, husk and grains etc. and after digestion it has been used for ICP-MS analysis. The crushed sample of shoot, root and grain were also used for synchrotron based XRF analysis after making its pellet. Apart from this, leave's xylem-phloem internal integrity was also assessed under field emission scanning electron microscope (FE-SEM).

\section{RESULTS AND DISCUSSIONS}

\subsection{Arsenic (As) concentration in rice grains}

In Aman (monsoon), 2016, the concentration of As (all in $\mathrm{m} \mathrm{kg}^{-1}$ ) in rice grains in Gosai, Satabdi, Gosai 


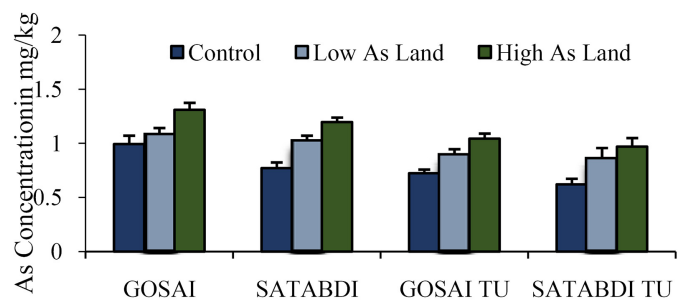

Two Cultivars With/Without TU (Thiourea)

Figure 1. Arsenic (As) concentration in $\mathrm{mg} \mathrm{kg}^{-1}$ in two cultivars of rice with and without thiourea (TU). All values are means of triplicates $\pm \mathrm{SD}$.

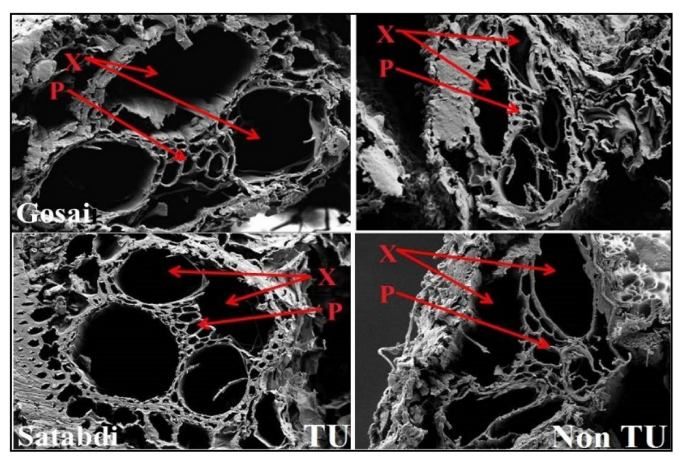

Figure 2. FE-SEM images of differentiated xylem (X) and phloem $(\mathrm{P})$ integrity in TU amended and non-TU rice plant leaf of both cultivars.

(TU) and Satabdi (TU) were found 1.086, 1.030, and $0.900,0.866$, respectively in low As, $1.31,1.196,1.043$ and 0.97 , respectively in high As and 0.994, 0.77, $0.723,0.62$, respectively in control (Fig. 1). The accumulation of As was thus significantly reduced in grains under TU supplementation.

\subsection{Growth and yield parameters}

The growth and yield were also improved in two cultivars under TU supplementation as compared to without TU plants. The biomass, tiller no., leaf area were increased in rice plants. The yield of rice grains was also increased in TU ameliorated cultivars in all the fields (Table 1).
Table 1. This table shows the yield of rice in $\mathrm{kg}$.

\begin{tabular}{llllll}
\hline & \multicolumn{2}{l}{ Satabdi (IET-4786) } & & \multicolumn{2}{l}{ Gosai } \\
\cline { 6 - 7 } \cline { 6 - 7 } Setting & TU & NON TU & & TU & NON TU \\
\hline Control field & 14.4 & 12.9 & & 14.0 & 13.8 \\
Low As field & 16.1 & 15.4 & & 15.3 & 13.5 \\
High As field & 12.9 & 12.6 & & 12.1 & 11.4 \\
\hline
\end{tabular}

\subsection{Alteration in integrity of xylem/phloem}

Internal organs (xylem phloem, bundle sheath) $\mathrm{OF}$ rice leaves integrity were distorted due to As toxicity. TU actually improved the vascular organ's integrity (Fig. 2). So, this could be a possible reason behind better biomass growth and yield in rice.

\section{CONCLUSIONS}

This study indicates that TU can reduce the As load in rice grain, which is a significant result. Further, TU supplementation also improved growth of plants along with yield. Hence, TU supplementation through seed soaking and spraying at the time of flowering can be a feasible strategy to mitigate As toxicity through reduce its translocation to grains in contaminated areas and grow rice with low arsenic in rice grains.

\section{ACKNOWLEDGEMENTS}

The authors are thankful to IESD-BHU, Varanasi, India for the lab facility. We are thankful to core instrumentation facilities of IISER-Kolkata, Mohanpur. MKU is thankful to UGC-BHU for Ph. D fellowship.

\section{REFERENCES}

Kelner, M.J., Bagnell, R. \& Welch, K.J. 1980. Thioureas react with superoxide radicals to yield a sulfhydryl compound. Explanation for protective effect against paraquat. J. Biological Chemistry 265(3): 1306-1311.

Srivastava, S. \& D'souza. S.F. 2009. Increasing sulfur supply enhances tolerance to arsenic and its accumulation in Hydrilla verticillata (L.f.) Royle. Environmental Science \& Technology 43(16): 6308-6313. 